\title{
Invited review: Application of meta-omics to understand the dynamic nature of the rumen microbiome and how it responds to diet in ruminants
}

\author{
R. J. Gruninger ${ }^{1, \dagger}$, G. O. Ribeiro ${ }^{2}$, A. Cameron ${ }^{1}$ and T. A. McAllister ${ }^{1}$ \\ ${ }^{1}$ Lethbridge Research and Development Centre, Agriculture and Agri-Food Canada, Lethbridge, Alberta T1J 4B1, Canada; ${ }^{2}$ Department of Production Animal Health \\ Faculty of Veterinary Medicine, University of Calgary, Calgary, Alberta T2N 4Z6, Canada
}

(Received 15 July 2018; Accepted 31 January 2019; First published online 7 May 2019)

\begin{abstract}
Ruminants are unique among livestock due to their ability to efficiently convert plant cell wall carbohydrates into meat and milk. This ability is a result of the evolution of an essential symbiotic association with a complex microbial community in the rumen that includes vast numbers of bacteria, methanogenic archaea, anaerobic fungi and protozoa. These microbes produce a diverse array of enzymes that convert ingested feedstuffs into volatile fatty acids and microbial protein which are used by the animal for growth. Recent advances in high-throughput sequencing and bioinformatic analyses have helped to reveal how the composition of the rumen microbiome varies significantly during the development of the ruminant host, and with changes in diet. These sequencing efforts are also beginning to explain how shifts in the microbiome affect feed efficiency. In this review, we provide an overview of how meta-omics technologies have been applied to understanding the rumen microbiome, and the impact that diet has on the rumen microbial community.
\end{abstract}

Keywords: herbivore, metagenome, metatranscriptome, feed digestion, microbiology

\section{Implications}

Meta-omics is playing an increasingly important role in defining the ecology of the rumen ecosystem and its responses to changes in diet. Metatranscriptomics is shedding light on rumen function and gaging the contribution of microbiota to important ruminal metabolic processes such as carbohydrate and protein digestion. Emerging long-read sequencing technologies are sure to advance our present understanding of interrelationships among microbes and their genes. Application of these technologies in a manner that focuses on host - rumen microbiome interactions is the key to further advancing our understanding of this fascinating ecosystem.

\section{The rumen and the rumen microbiome}

Ruminants evolved approximately 50 million years ago and are among the most widely adapted large mammals on earth, with an estimated 200 species inhabiting environments from the arctic to the tropics (Hackmann and Spain, 2010). They are unique among livestock because they can efficiently utilize forages, food by-products and non-protein nitrogen to

\footnotetext{
† E-mail: robert.gruninger@canada.ca
}

produce milk and meat, thereby avoiding plant materials more suitable for human consumption. Like all mammalian herbivores, ruminants do not produce cellulolytic or hemicellulolytic enzymes to degrade ingested plant material. Instead, they rely on symbiotic associations with bacteria, fungi and protozoa within the rumen to perform this function. The rumen microbiome comprises bacteria (up to $10^{11} \mathrm{cell} / \mathrm{s} / \mathrm{ml}$ ), protozoa $\left(10^{4}\right.$ to $10^{6}$ cells $\left./ \mathrm{ml}\right)$, fungi $\left(10^{3}\right.$ to $10^{6}$ zoospores $\left./ \mathrm{ml}\right)$, methanogens $\left(10^{6}\right.$ cells $\left./ \mathrm{ml}\right)$ and bacteriophages $\left(10^{7}\right.$ to $10^{10}$ particles/ml) (Morgavi et al., 2013). These microbial symbionts are highly specialized in degrading lignocellulosic biomass, and the ruminant host is dependent on the array of enzymes produced by the microbial community to convert complex fibrous substrates into fermentable saccharides. Ultimately, sugars released from plant material are fermented by rumen bacteria and converted primarily into the volatile fatty acids (VFAs), acetate, propionate and butyrate. These VFAs along with microbial protein are utilized by the ruminant for maintenance, growth and lactation (Morgavi et al., 2013).

\section{Composition of the rumen microbiome}

Great efforts have been made to understand the composition of the rumen microbial community and how it changes in 
response to a variety of selective pressures (Huws et al., 2018). Initial studies involved culturing rumen bacteria directly associated with the digestion of cellulose/hemicellulose and generated a substantial body of knowledge on the biology of the principal cellulolytic bacteria, Fibrobacter succinogenes, Ruminococcus albus, Ruminococcus flavefaciens and Butyrivibrio fibrisolvens (Morgavi et al., 2013) and the rumen fungi (Edwards et al., 2007). Far less is known about the rumen virome, and only recently has genomics shed light on the possible role of viruses within the rumen microbiota (Anderson et al., 2017; Gilbert et al., 2017). Although bacteria are better characterized, only $15 \%$ of the total species have been cultured in the laboratory (Morgavi et al., 2013; Creevey et al., 2014). Currently, there are at least 70 rumen bacterial species available in pure culture from public repositories (Creevey et al., 2014). Recent large-scale projects such as the Global Rumen Census (Henderson et al., 2015) and the Rumen Microbial Genomics Network Hungate1000 project (Seshadri et al., 2018) have enhanced our understanding of the rumen microbial ecosystem and its role in fibre digestion. The Hungate1000 project sought to produce a reference set of rumen microbial genome sequences from cultivated rumen bacteria, methanogens, anaerobic fungi and ciliate protozoa. The project successfully generated a catalogue of 480 bacterial and 21 archaeal genomes but failed to generate complete genomes for either fungi or protozoa. The AT-rich nature of these eukaryotic genomes presents significant assembly challenges, but progress is being made in characterizing the functional genome of anaerobic fungi (Solomon et al., 2016; Haitjema et al., 2017; Gruninger et al., 2018).

In addition to studies involving pure cultures, the application of cultivation-independent methods including metagenomics, metatranscriptomics and metaproteomics has greatly enhanced the scientific communities' understanding of the structure and function of the rumen microbial ecosystem (Ribeiro et al., 2016). These cultivation-independent studies suggest that many of the microbes and carbohydrateactive enzymes (CAZymes) involved in lignocellulose digestion in the rumen are uncharacterized (Hess et al., 2011). One tool that has seen success in exploring the functions of these uncharacterized microbes is the assembly of bacterial and archaeal genomes from metagenomic sequence data from the rumen (Svartström et al., 2017; Stewart et al., 2018a). This method has been rapidly expanding the database of rumen microbial genome sequences. Despite the utility of this approach, it is imperative that the function of these genomes be confirmed through culturing and metabolomic confirmation. Continued efforts at exploring the microbial diversity present in the rumen could prove to be a valuable source of novel enzymatic activities and metabolic pathways that have applications for improving the feed conversion of ruminants and industrial biomass conversion with a focus on recalcitrant plant cell walls. Differentiating dietary conditions where this diversity contributes to improved feed efficiency from when it does not could be the key to obtaining consistent improvements in ruminal feed efficiency.
The Global Rumen Census described the composition of the rumen and camelid foregut bacterial and archaeal communities (i.e., 742 samples from 32 animal species across 35 countries) and how they were influenced by diet, host species and geography (Henderson et al., 2015). This project found that the rumen microbiome was significantly influenced by host species and diet, with diet being the most influential factor. The taxonomic identity of the major microbial genera was similar, but large differences in the relative abundance of these bacteria were observed. This study highlights the adaptability of the rumen microbiota and agrees with an earlier work that found that the majority of the variation in the gut microbial community of mammals can be attributed to differences in diet (Ley et al., 2008). Variation in the physical and chemical composition of the diet is believed to provide unique ecological niches that favour the selection of specific microbes (Henderson et al., 2015). The rumen microbial community works in a complex and interlinked manner. Changes in the diet of the host promotes changes in ruminal microbial metabolism, altering the production of VFAs and methane, and ultimately impacting meat and milk production (de Menezes et al., 2011).

The Global Rumen Census also found that the rumen microbial ecosystem is dominated by a core community composed of poorly characterised microbes (Henderson et al., 2015). The community was highly diverse at the level of the operational taxonomic unit (OTU) but, $89.4 \%$ of all sequences could be classified to just 30 bacterial groups that were found in over $90 \%$ of the samples (Henderson et al., 2015). The core bacterial OTUs belonging to Prevotella, Butyrivibrio, Ruminococcus and the unclassified Lachnospiraceae, Ruminococcaceae, Bacteroidales and Clostridiales were the most abundant bacterial groups in the rumen $(67.1 \%$ of all bacterial sequence data). A greater abundance of unclassified Bacteroidales and Ruminococcaceae were observed in the rumen of animals fed high-forage diets, and greater abundance of Prevotella and unclassified Succinivibrionaceae were observed in the rumen of animals fed diets containing concentrate. The common rumen bacteria Prevotella ruminicola, P. brevis, $P$. bryantii and $P$. albensis tended to be more abundant in high-concentrate diets, and Fibrobacter abundance was higher in forage-fed cattle (Henderson et al., 2015). In addition, higher microbial diversity is also observed in forage diets as compared to highconcentrate diets (Tapio et al., 2017), a finding that likely reflects the greater complexity of carbohydrates and possibly functional conditions (i.e., pH, rate of passage, osmolality) in ruminants fed forage-based diets.

Recent efforts using network analysis to examine the metabolic function of rumen microbial communities found that distinct taxa can have similar metabolic networks and perform similar metabolic processes (Taxis et al., 2015). This redundancy was postulated to be due to similarity in the metabolic inputs and outputs between different taxa. The significance of the high metabolic redundancy present in the rumen is not known, but it has been suggested that it plays a role in carbohydrate digestion (Weimer, 2015). All -omics-based methods rely heavily on well-annotated databases to assign 
function to genes/proteins. A recent study of the metabolic pathways present in the genomes of a number of rumen bacteria identified several non-standard metabolic pathways that are not adequately represented in current databases (Hackmann et al., 2017). Hackmann and colleagues found that the recognized pathways for metabolizing pentose and hexose sugars to short-chain fatty acids do not adequately explain the fermentation products generated by a wide range of rumen bacteria (Hackmann et al., 2017). They found that $44 \%$ of these bacteria encoded atypical metabolic pathways and identified several that are completely novel. This finding underscores the need to continue culturing rumen microbes to facilitate the development of a well-annotated, diverse sequence database that accurately represents the biological processes present in the rumen.

\section{Beyond bacteria: methanogenic archaea and eukaryotic members of the rumen microbiome community}

The rumen is also inhabited by archaea, fungi and protists. The archaea found in the rumen are exclusively methanogens, and these microbes primarily produce methane by the reduction of $\mathrm{CO}_{2}$ with $\mathrm{H}_{2}$ (Wang et al., 2017). The archaeal community is significantly less diverse than the bacterial community and was found to be similar across all regions of the world (Henderson et al., 2015). Methanobrevibacter gottschalkii and Methanobrevibacter ruminantium were the most abundant archaea and were found in almost all samples. The dominance of archaea within the genus Methanobrevibacter is consistent with the majority of previous studies examining the methanogen community in the rumen (Janssen and Kirs, 2008; Wang et al., 2017). Together with Methanosphaera sp. and two Methanomassiliicoccaceae-affiliated groups, five genera of methanogenic archaea comprised $89.2 \%$ of the community (Henderson et al., 2015). The recently named order Methanomassiliicoccales is phylogenetically related to Thermoplasmatales and has been referred to by a number of names including 'rumen cluster $C^{\prime}$, 'Thermoplasmatales affiliated lineage $C^{\prime}$ ', or 'order Methanoplasmatales' (Borrel et al., 2014; Wang et al., 2017). This group of methanogenic archaea is commonly found in the rumen, and in some ruminants have been found to make up a significant proportion of the archaeal community (Wang et al., 2016b).

Much less is known about the ecology of the eukaryotic component of the rumen microbial community, namely the rumen fungi (Phylum Neocallimastigomycota) and the ciliated rumen protists (Groups Isotricha and Eudiplodinium). The anaerobic fungi play a key role in the initial colonization and degradation of the plant cell wall through the concerted action of carbohydrate-active enzymes and physical penetration of the plant cell wall by hyphae (Edwards et al., 2007). There are currently 11 cultured anaerobic fungi; however, metagenomic studies targeting the Internal transcribed spacer 1 (ITS-1) amplicon have revealed much greater taxonomic diversity in the rumen fungi with at least seven more phylogenetically distinct clades that are not represented by cultured isolates (Liggenstoffer et al., 2010; Koetschan et al., 2014; Tapio et al., 2017). Rumen fungi are associated with forage-based diets, and their abundance decreases rapidly upon the addition of concentrate to the diet (Belanche et al., 2012). In 2011, the first metatranscriptomic study of the rumen sequenced transcripts from the eukaryotic members of the rumen microbial community (Qi et al., 2011). In this seminal study, Qi and colleagues found that rumen eukaryotes contributed a significant portion of diverse cellulases, hemicellulases and esterases and that these proteins showed low levels of sequence identity to characterized proteins. In addition, many of these enzymes belonged to carbohydrate-active enzyme families that are not found in bacteria (Qi et al., 2011).

Advances in sequencing technology and bioinformatics tools have enabled researchers to sequence the genomes of four species of Neocallimastigomycota (Youseff et al., 2013; Haitjema et al., 2017). All of these genome assemblies are fragmented to varying degrees. Despite this challenge, these genome sequences have revealed valuable insight into the evolution of anaerobic fungi and the adaptations they have acquired to survive in a competitive anaerobic environment. Some of these adaptations are the presence of tetrahymanol in the plasma membrane instead of ergesterol, pyruvate metabolism occurring via mixed acid fermentation (Youssef et al., 2013) and the use of hydrogenosomes for ATP generation (Yarlett et al., 1986). The existence of cellulosomes in Neocallimastigomycota has long been proposed, but until recently the identity of the scaffoldin protein was not known. Using a combination of genomics and proteomics, the scaffoldin in Neocallimastigomycota was identified, and the dockerin-scaffoldin interaction has been examined (Haitjema et al., 2017). Unlike the high-specificity dockerinscaffoldin interaction in bacterial cellulosomes, the anaerobic fungal scaffoldin showed cross-reactivity between dockerincontaining proteins from multiple species of anaerobic fungi. This feature of the fungal cellulosome may be important for the co-existence of multiple species of fungi within the rumen (Haitjema et al., 2017). More recently, a transcriptomic analysis of carbohydrate metabolism in diverse species of anaerobic fungi found that the carbohydrate-active enzymes expressed by several species of rumen fungi may preferentially target different carbohydrate components within the plant cell wall (Gruninger et al., 2018). It was suggested that this is another mechanism that may decrease the direct competition for resources and facilitate the co-existence of multiple species within the rumen (Gruninger et al., 2018). These genomic (Youssef et al., 2013; Haitjema et al., 2017) and transcriptomic (Solomon et al., 2016; Henske et al., 2017; Gruninger et al., 2018) studies of rumen fungi have yielded valuable insight into the biology of these microbes and found that a core set of genes and diverse array of plant cell welldegrading enzymes have been maintained throughout the evolution of the phylum Neocallimastigomycota. These features likely facilitate the survival of these microbes in the competitive rumen environment. 

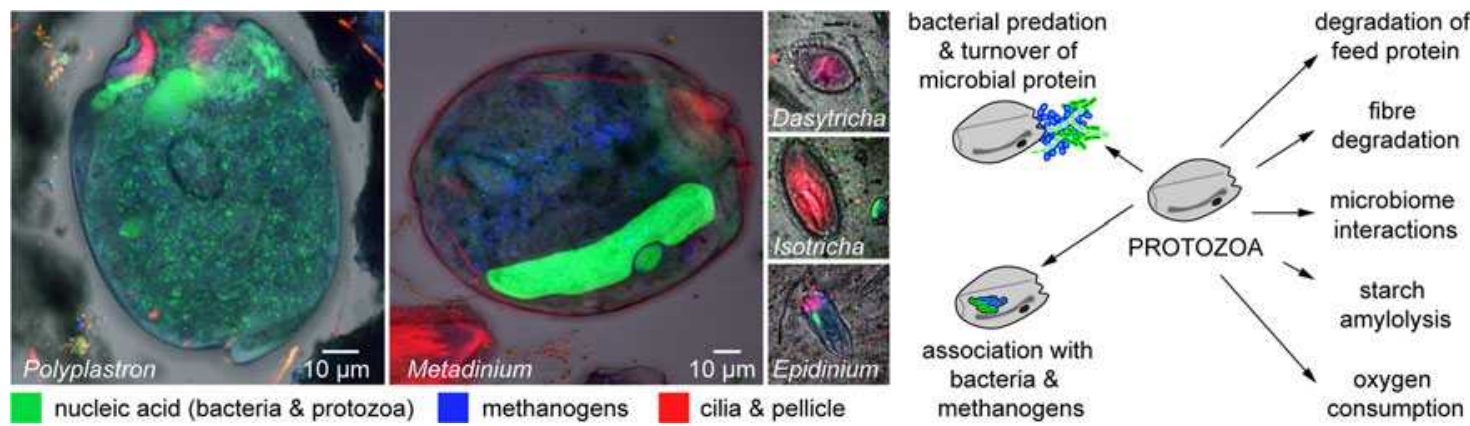

Figure 1 (colour online) Microbes associated with the rumen protozoa Polyplastron and Metadinium. Note that microbiomes associated with feed particles are also visible in the lower- and upper-left regions of the image with Polyplastron. Microbiomes can consist of complex or simple communities as illustrated by the almost-exclusive colonization of the outer surface of Metadinium by methanogens. Protozoa also perform a number of important functions within the rumen microbiome community. Blue bar $=10 \mu \mathrm{m}$. Protozoal samples were stained and visualized as described by Valle et al. (2015)

It has been proposed that ciliate protozoa may account for up to $50 \%$ of the rumen microbial biomass (Hungate et al., 1971), but more recent studies that have measured protozoal volume using video microscopy have suggested that it may be far lower (Wenner et al., 2018). The role of protozoa within the rumen microbiome is considered controversial (Newbold et al., 2015). Studies with protozoa-free ruminants have demonstrated that they are not absolutely essential for rumen function. Protozoa are highly proteolytic and through bacterial predation are responsible for the turnover of a large portion of the microbial protein within the rumen (Figure 1). Protozoa also contribute to the degradation of feed protein and are associated with higher ruminal concentration of ammonia nitrogen. A myriad of protozoa eradication or removal strategies have been developed because of the association these microbes have with decreased efficiency of rumen microbial protein synthesis, and increased methane emissions (Guyader et al., 2014; Newbold et al., 2015). The rumen ciliates associated with methane production can harbour both epi- and endo-symbiotic methanogens which derive hydrogen from hydrogenosome organelles within these protozoa (Ellis et al., 1994). Eliminating protozoa from the rumen has proven difficult, and an effective treatment or feed additive is not currently commercially available. Contrarily, it has also been shown that protozoa can also have positive effects on ruminal feed digestion (Huws et al., 2018). Furthermore, protozoa can rapidly engulf starch granules when animals are fed high-grain diets, competing with ruminal amylolytic bacteria for substrate and reducing the rate of starch fermentation. Such events can slow down the rate of starch fermentation, possibly reducing the risk of ruminal acidosis (Owens et al., 1998; Newbold et al., 2015). In high-forage diets rumen protozoa have been estimated to account for $17 \%$ to $21 \%$ of total fibre degradation (Dijkstra and Tamminga, 1995). Studies in defaunated sheep fed forage diets showed that fibre digestibility declined by $14 \%$ to $17 \%$ (Belanche et al., 2011, 2015). Protozoa also directly aid rumen fibre degradation through their involvement in the initial stages of fibre colonization and production of glycosyl hydrolases (GHs), as well as indirectly, through their consumption of low concentrations of $\mathrm{O}_{2}$ that can enter the rumen and destabilize ruminal metabolism
(Hillman et al., 1985; Ellis et al., 1989; Findley et al., 2011; Newbold et al., 2015). Protozoa also have complex interactions with other rumen microbes: a meta-analysis of defaunated ruminants indicated that they possessed fewer fibrolytic microbes, including anaerobic fungi (decreasing by $92 \%$ ), $R$. albus (decreasing by $34 \%$ ) and $R$. flavefaciens (decreasing by 22\%) (Newbold et al., 2015). However, the lack of sequenced genomes for rumen protozoa has made advancements in the understanding of their role in ruminal degradation of carbohydrates and proteins using 'meta-omic' approaches difficult (Comtet-Marre et al., 2017).

\section{Studying the rumen microbiome with -omics approaches}

Until recently, the rumen microbiome was primarily studied using culture-based or classical molecular techniques, including restriction fragment length polymorphism (RFLP), denaturing gradient gel electrophoresis (DGGE) and ribosomal RNA clone libraries. These molecular techniques have become largely obsolete, as the complexity of the rumen microbiome makes approaches such as metagenomics, metatranscriptomics and metaproteomics more meaningful for the study of the rumen microbiome. Amplicon-based metagenomic studies, also known as metataxonomics, sequence regions of marker genes such as the $16 \mathrm{~S} r R N A$, methyl coenzyme $\mathrm{M}$ reductase A (mcrA), 185 rRNA or ITS-1 that can be used to describe the bacterial, archaeal, protozoal and fungal populations, respectively (Li et al., 2016). These studies provide information on the composition of the microbial community, but little information about their function (Dai et al., 2015; Shinkai et al., 2016; Comtet-Marre et al., 2017). In contrast, shotgun metagenomics, metatranscriptomics and metaproteomics can provide a snapshot of both the taxonomic composition and the metabolic activity of a rumen microbial community under a particular set of conditions that dictate function. Metagenome-based approaches tend to be biased towards numerically abundant genes harboured by the most abundant microbial species. Increasingly, shotgun metagenomics is being used to reconstruct whole genomes of uncultured rumen microbes, providing novel insight into 
the biological mechanisms they have evolved to let them thrive in the rumen (Hess et al., 2011; Svartström et al., 2017).

Trying to understand a highly complex community such as the rumen using a single approach leads to the development of an incomplete picture. To address this limitation, a greater number of researchers are using a multi-omics approach combining metagenomics, metatranscriptomics, metaproteomics and metabolomics to study the rumen (Shinkai et al., 2016; Comtet-Marre et al., 2017; Deusch et al., 2017). In the study of the rumen, combining the results of metatranscriptomics and metagenomics has provided novel insights into the specific microbes active in lignocellulose digestion (Qi et al., 2011; Dai et al., 2015; Li and Guan, 2017). A metatranscriptome study of dairy cows on a forage-based diet found that the most-expressed CAZymes in the rumen were GH5, GH9, GH45 and GH48, primarily from Ruminococcus and Fibrobacter (Dai et al., 2015). The metagenomic study of rumen contents from dairy cows fed a high-forage diet by Hess and colleagues likewise found that the GH5 and GH9 CAZyme families putatively involved in cellulose digestion were abundant, whereas GH45 and GH48 were not (Hess et al., 2011). Differences in gene abundances observed between DNA- or RNA-based studies have been reinforced by several other rumen metatranscriptome studies, thus highlighting the importance of combined approaches (Qi et al., 2011; Shinkai et al., 2016; ComtetMarre et al., 2017). These studies have pointed to the rumen fungi, Prevotella sp., Ruminococcus sp. and Fibrobactersp. as being responsible for the production of a large proportion of the rumen CAZymes involved in lignocellulose digestion (Dai et al., 2015; Shinkai et al., 2016; Comtet-Marre et al., 2017).

Metagenomics and metatranscriptomics have also been used to examine the link between feed efficiency (based on residual feed intake (RFI)) and the rumen microbiome (Shabat et al., 2016; Li and Guan, 2017). With both approaches it was found that cattle with low feed efficiency have a greater diversity of metabolic pathways than those that are more efficient (Li and Guan, 2017). Increasing the diversity of metabolic pathways present in the rumen seems to increase the likelihood of carbon being shuttled into pathways that are less energy efficient, reducing the amount of energy the host can derive from metabolism. In contrast, highly efficient animals seem to have microbial communities that direct more carbon into fewer, more efficient metabolic pathways which lower the production of less metabolically valuable end products, such as methane (Shabat et al., 2016; $\mathrm{Li}$ and Guan, 2017). Both of these studies included a total mixed ration (TMR) containing significant levels of concentrate so it would be interesting to examine whether this relationship holds in cattle fed a diet with high forage and little to no concentrate. We recently conducted a study with cattle fed a $70 \%$ barley straw diet and observed apparent neutral detergent fibre digestibility ranging from $42.2 \%$ to $61.1 \%$ (Ribeiro et al., 2017). Fibre digestibility in this study was highly correlated with Metadinium protozoa. This suggests that there is high variability in the ability of individual cattle to digest recalcitrant fibre, and that more work should be done towards defining the role of protozoa and fungi in this process. The studies conducted to date suggest that lower microbial diversity in the rumen is linked to higher feed efficiency. It is not known how this might influence other important aspects of the animal, including adaptation to diet changes, resilience to illness or recovery from acidosis, and further research into examining the link between feed efficiency, the rumen microbiome, and the host is warranted.

Applying a combination of metagenomics and metatranscriptomics has enabled researchers to link members of the rumen microbiome in sheep that differ in methane production levels to rumen VFA profiles in these animals (Franzosa et al., 2014; Kamke et al., 2016). In another study, a systems biology approach using $16 \mathrm{~S}$ rRNA amplicon sequencing, metaproteomics and metabolomics found that the starch content of different forage-based diets accounted for an increase in the abundance and metabolic activity of bacteria in the family Succinivibrionaceae, particularly starch-utilizing Succinionas amylolytica and Ruminobacter amylophilus (Deusch et al., 2017). Further efforts are required in this area to link shifts in microbiome composition and biological activity to host phenotype and host physiology. Another area requiring further efforts to better understand is how the rumen microbiome changes over time. Many -omics studies of the rumen to date have only examined samples taken during a limited time scale. There is a need for longitudinal studies to examine how the microbiome changes over time as temporal changes also influence the development of the microbiome and ultimately its impact on host productivity (Shaani et al., 2018).

\section{Technical challenges of sequence-based studies of the rumen}

It is well known that sequencing different variable regions of the $16 \mathrm{~S}$ rRNA gene influences the taxonomy assigned to those sequences; this is one of the most common sources of variation between microbiome studies (Pollock et al., 2018). Unfortunately, there is still no clear consensus as to which variable region of the most commonly used amplicon, the 16S rRNA gene, provides the greatest classification accuracy (Claesson et al., 2010; D'Amore et al., 2016; Yang et al., 2016). The most commonly used region for Illumina-based sequencing is the V4 region (Caporaso et al., 2012). Sequencing the 16S rRNA V4 region has consistently shown the highest similarity to expected taxonomic distribution, and gives results similar to those obtained from shotgun metagenomics, a sequencing approach not subject to PCR bias (Caporaso et al., 2012; Tremblay et al., 2015; D'Amore et al., 2016).

The recent focus on using short-read technologies to characterize microbial communities has resulted in less fulllength 165 rRNA sequences. Full-length sequences improve the depth of phylogenetic analyses and are useful for the design of lineage-specific PCR primers and fluorescent in situ hybridization (FISH) probes (Schloss et al., 2016). To address this limitation, PacBio SMRT long-read technology has been used to generate full-length 165 rRNA sequences with error rates of $0.027 \%$ to $-0.69 \%$ : rates comparable to Illumina 
technology. Shorter reads from Illumina sequencing can characterize microbial communities at the OTU level but are less accurate at characterizing communities at the genus and species level (Liu et al., 2007; Soergel et al., 2012). Myer et al. (2016) compared whether full-length 16S rRNA reads (V1-V8) sequenced using PacBio were able to classify rumen community members at greater depth than shorter V1-V3 reads sequenced using Illumina Mi-Seq. They found that while the two platforms revealed similar microbial OTUs, species richness, Good's coverage and Shannon diversity metrics, the Pac-Bio improved the taxonomic depth. Adoption of Oxford Nanopore MinION will undoubtedly accelerate the use of full-length sequencing in the generation of metagenomic assemblies, as it has already shown promise as a tool to assemble whole chromosomes from complex rumen metagenomes (Stewart et al., 2018b).

Several factors can generate variation between metagenomics studies, including sampling method, sampling site, number of samples, library preparation (Clooney et al., 2016), sequencing technology (D'Amore et al., 2016), differences in bioinformatic pipelines (Pollock et al., 2018), differences in databases (Soergel et al., 2012; Schloss et al., 2016) and even sequencing facility (Kim and Yu, 2014). It is also well known that the microbiome among individuals varies even when other factors such as diet composition are kept constant (Flores et al., 2014). Therefore, researchers should keep in mind that comparisons of relative abundances of OTUs and taxa between different studies can, in principle, lead to erroneous conclusions. This is particularly the case for OTUs or taxa that account for a relatively low percentage of the bacterial population (Kim and Yu, 2014).

\section{Development of the rumen microbiome in young ruminants}

A number of studies have examined the development of the rumen microbiome from neonate to the mature animal (Jami et al., 2013; Jiao et al., 2016; Meale et al., 2016; Wang et al., 2016a). Shortly after birth, there is a shift in the bacterial community from a highly variable aerobic and facultative anaerobic community to a diverse community of mainly obligate anaerobes (Jami et al., 2013). This transition climaxes at approximately 3 months of age and is characterized by an increase in the abundance of Bacteroidetes and a decrease in the abundance of Proteobacteria (Jami et al., 2013; Jiao et al., 2016; Wang et al., 2016a). Within the phylum Firmicutes, Bacteroides is the most abundant genus in newborn calves, whereas in 2-month-old calves, Prevotella is the principal genera observed (Jami et al., 2013). This microbial succession was linked to alterations in the diet associated with a shift from a primarily milk-based diet to a post-weaning forage-based diet (Jami et al., 2013; Meale et al., 2016; Wang et al., 2016a). Undoubtedly, changes in diet and feeding behaviour also contribute to changes in rumen morphology and function and ultimately the rumen microbiome. For example, in nursing ruminants, a specialized structure called the reticular groove directs milk to flow into the abomasum, by-passing the rumen. Interestingly, the celluloytic bacteria Ruminococcus flavefaciens and $R$. albus were detected in the rumen of 1- and 3-day-old calves respectively, while Fibrobacter succinogenes was not detectable in the rumen until after 2 months of age (Jami et al., 2013). Archaeal, bacterial and fungal communities can be detected after 7 days in the rumen of calves that have only received milk, with populations varying thereafter with both age of the calf and the type of diet (Dias et al., 2017).

\section{Impact of forage on the rumen microbiome}

After weaning, the diet of the ruminant shifts to one primarily consisting of high-fibre forages and grains. The ability of ruminants to digest high-fibre forages is due to the enzymatic activity of the rumen microbiome. Alterations in the chemical composition of forage, the addition of concentrates or the addition of other additives can alter the composition of the rumen microbiome. One study examining the impact that differences in the composition of forage have on the rumen microbiome of dairy cows found that animals fed pasture or TMR diets did not have obvious differences at the phylum level, with Firmicutes and Bacteroidetes representing approximately $80 \%$ of the total rumen microbiome sequences (de Menezes et al., 2011). This did not hold true at higher levels of taxonomic resolution as there were clear differences between pasture and TMR diets for Bacteroidetes, Firmicutes, Fibrobacteres and Proteobacteria (de Menezes et al., 2011). Interestingly, the family Fibrobacteriaceae accounted for almost $10 \%$ of the sequences in solid rumen contents from cattle fed TMR diets compared to $3 \%$ in cattle on pasture. The observed increase in Fibrobacteriaceae was suggested to be due to an increased level of lignocellulose in the diet from the addition of straw ( $3 \%$ of diet DM) to the diet. This hypothesis is supported by Ribeiro et al. (2017) who found that Fibrobacteraceae accounted for $25 \%$ of the OTUs in rumen solids from heifers fed a $70 \%$ barley straw diet. In other studies, Fibrobacteriaceae were only a minor component in cattle fed TMR diets indicating that variability between animals likely exists (Callaway et al., 2010; Zened et al., 2013).

Looking specifically at the effect of forage source in the diet, Kong et al. (2010) observed that cows fed an alfalfa hay-based diet had different bacterial communities and greater species richness than cows fed a triticale straw-based diet. This seems to be related to the greater nutrient availability in alfalfa hay for ruminal microbial populations, as compared to the restricted nutrient content of triticale straw. Prevotella were the most abundant genera (comprising $14 \%$ to $22 \%$ of the total clones) in both diets, suggesting that this genus plays a fundamental role in the rumen ecosystem (Kong et al., 2010). Changing the forage source (e.g. corn silage, grass silage or grass hay) in a $48 \%$ forage: $52 \%$ concentrate diet also altered the ruminal bacterial population of Jersey cows (Deusch et al., 2017). A higher abundance of Proteobacteria and Succinivibrionaceae were observed 
in the rumen of cows fed corn silage, consistent with the higher content of non-fibre carbohydrates in this diet as compared to grass diets. In agreement with previous studies, Prevotellaceae was the most abundant family detected (Deusch et al., 2017). Seasonal changes in pasture quality throughout the year also promoted changes in the ruminal bacterial communities attached to solid digesta collected from dairy cows grazing rye-grass/clover pasture (Noel et al., 2017). Overall, the most abundant bacterial groups were uncharacterized genera in the order Clostridiales accounting for $22.9 \%$ of OTUs, followed by uncharacterized genera in the family Lachnospiraceae (12.2\%) and Butyrivibrio (10.2\%), Ruminococcus (7.6\%) and Prevotella (6.7\%). This study showed that the rumen bacterial community can adapt to relatively small changes in forage quality, and to changes in the forage:grain ratio. Although small seasonal changes in ruminal bacterial communities were observed, principal members of the community remained consistent, suggesting their central role in feed degradation regardless of changes in pasture quality (Noel et al., 2017).

The Prevotellaceae are one of the most abundant microbes in the rumen and are known to have an extensive metabolic repertoire that enables the utilization of a wide range of substrates. The cellulolytic rumen bacteria Ruminococcus flavefaciens, Ruminococcus albus and Fibrobacter succinogenes are most abundant when the diet is mainly forage, but changes in the type of forage in the diet do not seem to alter the relative abundance of these bacteria (Deusch et al., 2017). It has been suggested that these fibrolytic bacteria may be regarded as 'keystone' species and that their numbers alone do not clearly represent their contribution to rumen fibre digestion (Ze et al., 2013; Creevey et al., 2014). Recent metatranscriptomic analysis of the rumen contents of cattle fed a 50:50 forage:concentrate TMR supports the pivotal role of these well-characterized fibrolytic bacteria (Prevotella, Ruminoccocus and Fibrobacter) in fibre degradation (Comtet-Marre et al., 2017). Several studies have also found low levels of these well-known cellulolytic bacteria in cattle fed a high-forage diet (Creevey et al., 2014; Tapio et al., 2017). Interestingly, Tapio et al. (2017) observed that increased forage content resulted in an increase the abundance of many uncharacterized bacteria within the Clostridiaceae and Lachnospiraceae families. Similar results were also observed by Henderson et al. (2015) and Noel et al. (2017), which suggests that there are largely undescribed bacteria in the rumen that may have an important role in fibre degradation. Further research characterizing these unknown bacteria using approaches that include ultra deep sequencing, the use of single-cell genomics or increased efforts at culturing rumen microbes is needed to obtain a more complete understanding of fibre digestion in the rumen (Hosokawa et al., 2017; Huws et al., 2018).

\section{Colonization of feed in the rumen}

When feed enters the rumen, it is rapidly colonized by rumen microbes, and the digestion of the plant cell wall begins within minutes (Edwards et al., 2007; Edwards et al., 2008). Bacteria preferentially attach to damaged sites on the plant surface, whereas fungi are able to physically disrupt the ingested material via rhizoidal growth. Microbial attachment is absolutely essential for the development of the complex microbial populations required for feed digestion in the rumen and occurs via a multistep process: (1) displacement of the epiphytic microbiome by rumen microbes (time $<1 \mathrm{~h}$ ), (2) establishment of a primary colonizing community of generalist microbes that primarily metabolize accessible carbohydrates (time $1 \mathrm{~h}$ to $4 \mathrm{~h}$ ), (3) loss of some primary colonizers and selection of secondary colonizers specialized in digesting hemicellulose and cellulose (time $>4 \mathrm{~h}$; Figure 2) (Piao et al., 2014; Huws et al., 2016; Mayorga et al., 2016). Within this community are taxa including Butyrivibrio, Fibrobacter, Olsenella and Prevotella that do not change significantly in abundance during primary and secondary colonization (Huws et al., 2016; Liu et al., 2016). Populations of Prevotella peak on the surface of fibre within $1 \mathrm{~h}$ in the rumen (Huws et al., 2016). Prevotella sp. have been noted to be ubiquitous in the rumen environment and have a wide range of metabolic capabilities (Petri et al., 2013; Rubino et al., 2017). This metabolic flexibility likely explains the involvement of Prevotella in both primary and secondary colonization of feed as it can utilize soluble carbohydrates, pectins, proteins and hemicellulose (Huws et al., 2016). Populations of Clostridia (Pseudobutyrivibrio, Roseburia and Ruminococcus sp.) peak after Prevotella, perhaps due to their role in targeting cellulose (Piao et al., 2014; Rubino et al., 2017). In accordance with this model, it has been observed that little biomass is degraded during primary colonization, with the majority of degradation occurring after this (Piao et al., 2014; Huws et al., 2016). It has also been hypothesized that decreasing microbial richness during the shift from primary to secondary colonization is due to niche specialization of Clostridiales and Bacteroidales, which play a specific role in lignocellulose degradation during secondary colonization (Huws et al., 2016; Mayorga et al., 2016; Rubino et al., 2017).

The structure and chemical composition of feedstuffs is a key determinant defining which microbes participate in the ordered colonization of feed (Huws et al., 2014; Liu et al., 2016). The shifting nature of the community involved in feed digestion underlies the need to allow animals to adapt to shifts in diet, particularly from forage-based to concentratebased diets. This transition has been reported to require approximately 14 days and involves significant changes in ruminal microbial populations (Petri et al., 2013; Pitta et al., 2014; Kittelmann et al., 2015). Transition from a mainly forage diet to a high-concentrate diet has been shown in several studies to lead to an increase in the abundance of Bacteroidetes and a decrease in Firmicutes and Proteobacteria (Petri et al., 2013; Kittelmann et al., 2015). Inclusion of cereal grain in the diet results in higher levels of ruminal starch, which promotes the growth of amylolytic bacteria such as Ruminobacter sp. and Succinivibrio sp. (Kittelmann et al., 2015). In forage diets, there is a significant 


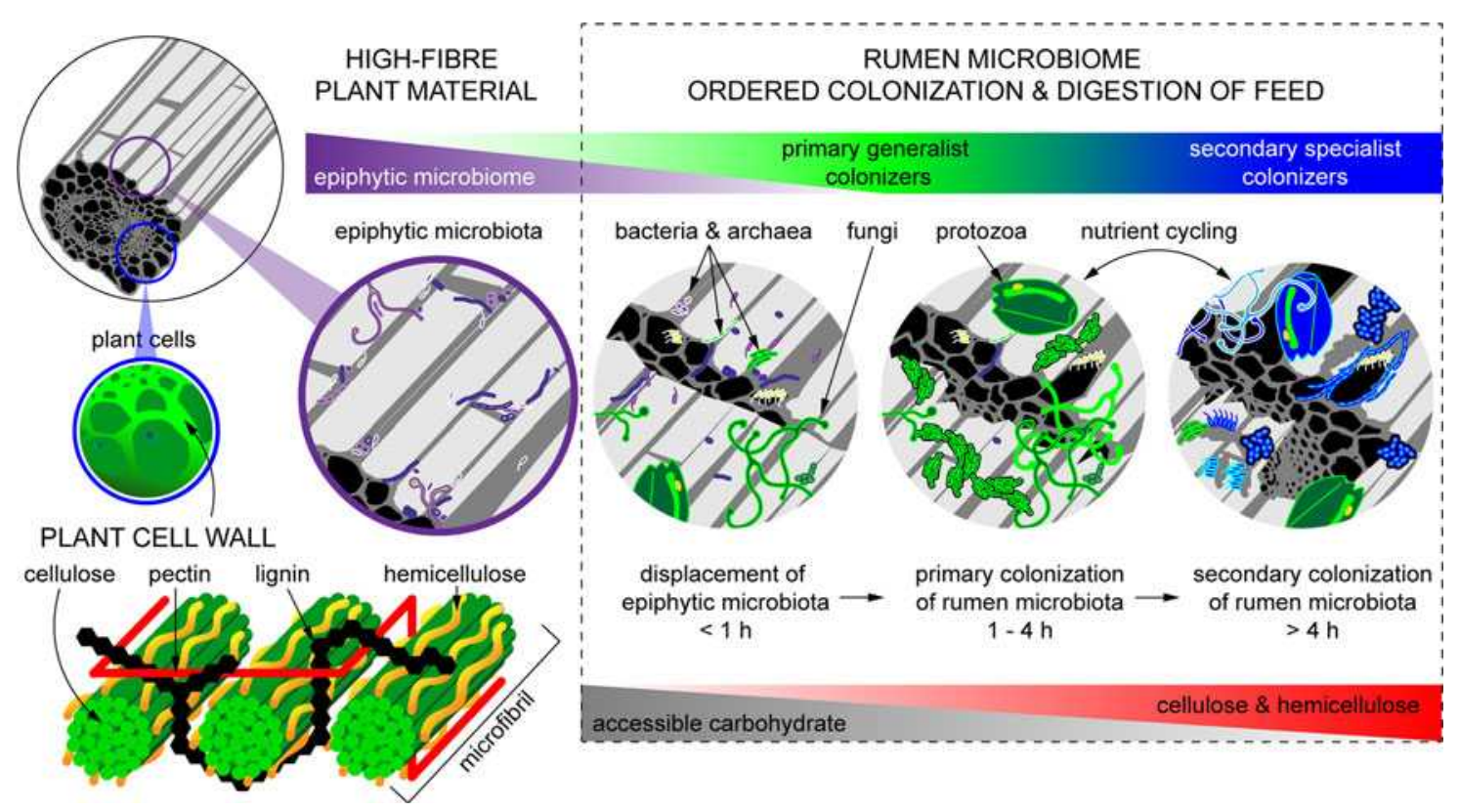

Figure 2 (colour online) Fibrous material in plants is colonized by a natural epiphytic microbiome which colonizes the forage while it grows in the field. This microbiome can be altered if the plant is ensiled prior to consumption. Upon consumption this epiphytic population is displaced by primary colonizing bacteria that ferment primarily sugars and soluble proteins. These primary colonizers are in turn replaced by secondary colonizers which play a more active role in the digestion of structural carbohydrates in plant cell walls.

increase in fibrolytic microbes, including anaerobic fungi, Ruminococcaceae, as well as non-fibrolytic bacteria such as Succiniclasticum sp. (Kumar et al., 2015). Anaerobic fungi are highly responsive to increases in concentrate levels, and their abundance decreases with increasing starch in the diet (Belanche et al., 2012). However, alterations in diet seem to have less impact on the abundance of methanogenic archaea (Kumar et al., 2015), possibly because of the more uniform availability of reducing equivalents in the rumen and their ability to avoid washout through physical associations such as those observed with protozoa (Levy and Jami, 2018).

\section{Role of diet in dysbioses of the rumen microbiome}

Rapid diet changes that do not enable the rumen microbes to adapt can lead to digestive upset and other health problems. For example, frothy bloat occurs in ruminants consuming a diet with high levels of rapidly digestible soluble protein and carbohydrate (Grilli et al., 2016). Bloat can be caused by abrupt shifts to protein-rich leguminous forages, as well as by rapid shifts to extremely high levels of concentrate. The inability of the animal to release gas produced by rumen fermentation due to impairment of eructation can prevent the contraction of the diaphragm and, if not relieved, lead to suffocation. Bloat in cattle grazing wheat pastures was linked to increased production of biofilm-associated mucopolysaccharide in the rumen resulting from diet-induced shifts in the rumen bacterial population (Min et al., 2006). Frothy bloat in goats resulted in long-lasting changes in the structure of the rumen microbial community, which persisted even after the clinical manifestations of bloat ceased (Grilli et al., 2016). A metagenomic study examining the microbial role in frothy wheat bloat in cattle revealed disruption in symbiotic relationships among microbial taxa, increased abundance of methanogenic archaea and a reduction in the abundance and diversity of CAZymes, suggesting an alteration in carbohydrate metabolism (Pitta et al., 2016).

Clinical (rumen $\mathrm{pH}<5.2$ ) and sub-clinical acidosis (rumen pH 5.2 to 5.6 for at least $3 \mathrm{~h}$ ) are conditions that can also be linked with shifts in the rumen microbiome (Owens et al., 1998). The classical view of acidosis is that rapid fermentation of feed results in the production of VFAs at a rate greater than they can be absorbed across the rumen wall, or pass through the rumen to the lower digestive tract. As a consequence, the $\mathrm{pH}$ of the rumen decreases to a point where cellulolytic bacteria are inhibited and acid-tolerant, lactateproducing bacteria, particularly Streptococcus bovis and Lactobacillus sp., predominate (Khafipour et al., 2009). These conditions are correlated with a reduction in species richness and diversity of the rumen microbiota (Khafipour et al., 2009; Petri et al., 2013; McCann et al., 2016; Plaizier et al., 2017). This can be modulated, however, by the activity of the lactic acid-utilizing bacteria Megasphaera elsdenii and Selenomonas ruminantium. The activity of these bacteria is increased at high lactate concentrations, and consequently, lactic acid concentrations often do not reach levels associated with clinical acidosis (McCann et al., 2016). Acidosis is also associated with higher levels of Escherichia coli in the rumen and production of ruminal lipopolysaccharides (LPS) which contributes to systemic inflammation in grain-fed cattle suffering from clinical acidosis (Plaizier et al., 2016). Indeed, profiling of the $E$. coli population in acidotic rumen contents revealed a shift in the population towards $E$. coli isolates with unique virulence factors that trigger inflammatory responses (Khafipour et al., 2011). Despite 
acidosis-associated changes in the rumen microbiome, the core microbial community remains seemingly unaltered, and recovery of the rumen microbiome has been shown to occur approximately 1 week following clinical or subclinical acidosis (Petri et al., 2013).

\section{Future opportunities}

Although great progress has been made in the past decade towards a better understanding of the rumen microbiome under a range of conditions, there is still a great deal that is not understood. Efforts must be made to continue to culture rumen bacteria that have to date resisted cultivation. The integration of genomic information from efforts such as Hungate1000 will hopefully enable researchers to design novel growth media with essential co-factors and energy sources that these uncultured organisms require. Although researchers have attempted to manipulate the rumen microbiome, the resiliency of the community has limited the success of such efforts (Weimer, 2015). We have recently attempted to manipulate the rumen of cattle by repeated inoculation with bison rumen contents (Ribeiro et al., 2017). Although initial changes in microbial population were observed, we were unsuccessful in improving fibre degradation. At present, it is unclear whether there is a point in the animal's life that the rumen microbiome becomes irreversibly programmed. Efforts towards a better understanding of the development of the rumen microbial community in young ruminants have provided some insights, but more needs to be done to determine if probiotics or other approaches can be used to generate a more resilient rumen microbial community that enhances the efficiency and health of the host.

Interactions between the host and the rumen microbiome are not well understood. Future studies need to not only consider interactions among microbes but also how microbial metabolites alter host gene expression in cells and tissues such as the rumen epithelium, liver and immune system. Recent work examining the connection between the immune system in ruminants has found host-specific interaction between salivary immunoglobulin IgA (Fouhse et al., 2017) and upregulation of several ruminal epithelial Toll-like receptors (TLRs) in response to high-grain diets (Liu et al., 2015). TLRs are known to be involved in recruitment of immune cells and the production of inflammatory cytokines. This hints at how changes in the rumen environment can cause systemic changes in the host and vice versa.

\section{Conclusion}

Over the past decade, significant research has been directed towards understanding both the composition of the rumen microbiome and how it affects the growth and health of the host. It is clear that diet can have dramatic effects on the taxonomic composition of the rumen, and that these changes are linked to the nature of the nutrients in the rumen. More efforts are needed to incorporate information on the nature of the microbes present and their metabolic activity through complementary -omics approaches. Efforts must now be made to understand how this community interacts with the physiological function of the rumen and the host, ultimately influencing both the health and productivity of the animal.

\section{Acknowledgements}

The authors wish to acknowledge the Beef Cattle Research Council of the Growing Forward II program of Agriculture and Agri-Food Canada for support of their work. We also wish to thank Edith Vale for generating the confocal microscopy pictures of rumen protozoa.

\section{Declaration of interest}

The authors have no conflicts to declare.

\section{Ethics statement}

This review did not require ethics approval.

\section{Software and data repository resources}

None of the data were deposited in an official repository.

\section{References}

Anderson CL, Sullivan MB and Fernando SC 2017. Dietary energy drives the dynamic response of bovine rumen viral communities. Microbiome 5, 155. doi: 10.1186/s40168-017-0374-3.

Belanche A, Abecia L, Holtrop G, Guada JA, Castrillo C, de la Fuente G and Balcells J 2011. Study of the effect of presence or absence of protozoa on rumen fermentation and microbial protein contribution to the chyme. Journal of Animal Science 89, 4163-4174.

Belanche A, de la Fuente $G$ and Newbold CJ 2015. Effect of progressive inoculation of fauna-free sheep with holotrich protozoa and total-fauna on rumen fermentation, microbial diversity and methane emissions. FEMS Microbiology Ecology 91, fiu026. doi: 10.1093/femsec/fiu026.

Belanche A, Doreau M, Edwards JE, Moorby JM, Pinloche E and Newbold CJ 2012. Shifts in the rumen microbiota due to the type of carbohydrate and level of protein ingested by dairy cattle are associated with changes in rumen fermentation. Journal of Nutrition 142, 1684-1692.

Borrel G, Parisot N, Harris HM, Peyretaillade E, Gaci N, Tottey W, Bardot O, Raymann K, Gribaldo S, Peyret P, O'Toole PW and Brugere JF 2014. Comparative genomics highlights the unique biology of Methanomassiliicoccales, a Thermoplasmatales-related seventh order of methanogenic archaea that encodes pyrrolysine. BMC Genomics 15, 679. doi: 10.1186/1471-2164-15-679.

Callaway T, Dowd S, Edrington T, Anderson R, Krueger N, Bauer N, Kononoff P and Nisbet D 2010. Evaluation of bacterial diversity in the rumen and feces of cattle fed different levels of dried distillers grains plus solubles using bacterial tag-encoded FLX amplicon pyrosequencing. Journal of Animal Science 88, 3977-3983.

Caporaso JG, Lauber CL, Walters WA, Berg-Lyons D, Huntley J, Fierer N, Owens SM, Betley J, Fraser L, Bauer M, Gormley N, Gilbert JA, Smith G and Knight R 2012. Ultra-high-throughput microbial community analysis on the Illumina HiSeq and MiSeq platforms. The ISME Journal 6, 1621-1624.

Claesson MJ, Wang Q, O'sullivan 0, Greene-Diniz R, Cole JR, Ross RP and OTtoole PW 2010. Comparison of two next-generation sequencing technologies for resolving highly complex microbiota composition using tandem variable $16 \mathrm{~S}$ rRNA gene regions. Nucleic Acids Research 38, e200. doi: 10.1093/nar/gkq873. 
Clooney AG, Fouhy F, Sleator RD, O'Driscoll A, Stanton C, Cotter PD and Claesson MJ 2016. Comparing apples and oranges?: next generation sequencing and its impact on microbiome analysis. PLoS One 11, e0148028.

Comtet-Marre S, Parisot N, Lepercq $P$, Chaucheyras-Durand $F$, Mosoni $P$, Peyretaillade E, Bayat AR, Shingfield KJ, Peyret P and Forano E 2017 Metatranscriptomics reveals the active bacterial and eukaryotic fibrolytic communities in the rumen of dairy cow fed a mixed diet. Frontiers in Microbiology 8, 67. doi: 10.3389/fmicb.2017.00067.

Creevey CJ, Kelly WJ, Henderson G and Leahy SC 2014. Determining the culturability of the rumen bacterial microbiome. Microbial Biotechnology 7, 467-479.

Dai X, Tian Y, Li J, Su X, Wang X, Zhao S, Liu L, Luo Y, Liu D and Zheng H 2015. Metatranscriptomic analyses of plant cell wall polysaccharide degradation by microorganisms in the cow rumen. Applied and Environmental Microbiology 81, 1375-1386.

D'Amore R, ljaz UZ, Schirmer M, Kenny JG, Gregory R, Darby AC, Shakya M, Podar M, Quince C and Hall N 2016. A comprehensive benchmarking study of protocols and sequencing platforms for 16S rRNA community profiling. BMC Genomics 17, 55. doi: 10.1186/s12864-015-2194-9.

de Menezes AB, Lewis E, O'Donovan M, O'Neill BF, Clipson N and Doyle EM 2011. Microbiome analysis of dairy cows fed pasture or total mixed ration diets. FEMS Microbiology Ecology 78, 256-265.

Deusch S, Camarinha-Silva A, Conrad J, Beifuss U, Rodehutscord M and Seifert J 2017. A structural and functional elucidation of the rumen microbiome influenced by various diets and microenvironments. Frontiers in Microbiology 8 1605. doi: $10.3389 /$ fmicb.2017.01605

Dias J, Marcondes M, Noronha MF, Resende RT, Machado FS, Mantovani HC, Dill-McFarland KA and Suen G 2017. Effect of pre-weaning diet on the ruminal archaeal, bacterial and fungal communities of dairy calves. Frontiers in Microbiology 8, 1553.doi: 10.3389/fmicb.2017.01553.

Dijkstra J and Tamminga S 1995. Simulation of the effects of diet on the contribution of rumen protozoa to degradation of fibre in the rumen. British Journa of Nutrition 74, 617-634.

Edwards JE, Huws SA, Kim EJ and Kingston-Smith AH 2007. Characterization of the dynamics of initial bacterial colonization of nonconserved forage in the bovine rumen. FEMS Microbiology Ecology 62, 323-335.

Edwards JE, Kingston-Smith AH, Jimenez HR, Huws SA, Skot KP, Griffith GW McEwan NR and Theodorou MK 2008. Dynamics of initial colonization of nonconserved perennial ryegrass by anaerobic fungi in the bovine rumen. FEMS Microbiology Ecology 66, 537-545.

Ellis J, Williams A and Lloyd D 1989. Oxygen consumption by ruminal microorganisms: protozoal and bacterial contributions. Applied and Environmental Microbiology 55, 2583-2587.

Ellis JE, Lindmark DG, Williams AG and Lloyd D 1994. Polypeptides of hydrogenosome-enriched fractions from rumen ciliate protozoa and trichomonads: immunological studies. FEMS Microbiology Letters 117, 211-216.

Findley SD, Mormile MR, Sommer-Hurley A, Zhang XC, Tipton P, Arnett K, Porte $\mathrm{JH}$, Kerley M and Stacey G 2011. Activity-based metagenomic screening and biochemical characterization of bovine ruminal protozoan glycoside hydrolases. Applied and Environmental Microbiology 77, 8106-8113.

Flores GE, Caporaso JG, Henley JB, Rideout JR, Domogala D, Chase J, Leff JW Vazquez-Baeza Y, Gonzalez A, Knight R, Dunn RR and Fierer N 2014. Temporal variability is a personalized feature of the human microbiome. Genome Biology 15, 531. doi: 10.1186/s13059-014-0531-y.

Fouhse JM, Smiegielski L, Tuplin M, Guan LL and Willing BP 2017. Host immune selection of rumen bacteria through salivary secretory IgA. Frontiers in Microbiology 8, 848. doi: 10.3389/fmicb.2017.00848.

Franzosa EA, Morgan XC, Segata N, Waldron L, Reyes J, Earl AM, Giannoukos G, Boylan MR, Ciulla D, Gevers D, Izard J, Garrett WS, Chan AT and Huttenhower C 2014. Relating the metatranscriptome and metagenome of the human gut. Proceedings of the National Academy of Sciences 111, E2329-E2338. doi: 10.1073/pnas.1319284111.

Gilbert RA, Kelly WJ, Altemann E, Leahy SC, Minchin C, Ouwerkerk D and Klieve A 2017. Toward understanding phage: host interactions in the rumen; complete genome sequences of lytic phages infecting rumen bacteria. Frontiers in Microbiology 8, 2340. doi: 10.3389/fmicb.2017.02340.

Grilli DJ, Mrázek J, Fliegerová K, Kopečný J, Lama SP, Cucchi MEC, Sosa MA and Arenas GN 2016. Ruminal bacterial community changes during adaptation of goats to fresh alfalfa forage. Livestock Science 191, 191-195.
Gruninger RJ, Nguyen TTM, Reid ID, Yanke JL, Wang P, Abott DW, Tsang A and McAllister T 2018. Application of transcriptomics to compare the carbohydrate active enzymes that are expressed by diverse genera of anaerobic fungi to degrade plant cell wall carbohydrates. Frontiers in Microbiology 9, 1581. doi: 10.3389/fmicb.2018.01581.

Guyader J, Eugene M, Noziere P, Morgavi DP, Doreau M and Martin C 2014. Influence of rumen protozoa on methane emission in ruminants: a meta-analysis approach. Animal 8, 1816-1825.

Hackmann TJ, Ngugi DK, Firkins JL and Tao J 2017. Genomes of rumen bacteria encode atypical pathways for fermenting hexoses to short-chain fatty acids. Environmental Microbiology 19, 4670-4683. doi: 10.1111/1462-2920.13929.

Hackmann TJ and Spain JN 2010. Invited review: ruminant ecology and evolution: perspectives useful to ruminant livestock research and production. Journal of Dairy Science 93, 1320-1334.

Haitjema CH, Gilmore SP, Henski JK, Solomon KV, de Groot AK, Mondo SJ, Salamov AA, LaButti K, Zhao Z, Chiniquy J, Barry K, Brewer HH, Purvine SO, Wright AT, Hainaut $M$, Boxma $B$, van Alen $T$, Hackstein JHP, Henrissat $B$, Baker SE, Grigoriev IV and O'Malley MA 2017. A parts list for fungal cellulosomes revealed by comparative genomics. Nature Biotechnology 2, 17087.

Henske JK, Gilmore SP, Knop D, Cunningham FJ, Sexton JA, Smallwood CR, Shutthanandan V, Evans JE, Theodorou MK and O'Malley MA 2017. Transcriptomic characterization of Caecomyces churrovis: a novel, nonrhizoid-forming lignocellulolytic anaerobic fungus. Biotechnol Biofuels 10, 305. Henderson G, Cox F, Ganesh S, Jonker A, Young W, Global Rumen Census Collaborators and Janssen PH 2015. Rumen microbial community composition varies with diet and host, but a core microbiome is found across a wide geographical range. Scientific Reports 5, 14567. doi: 10.1038/srep14567.

Hess M, Sczyrba A, Egan R, Kim T-W, Chokhawala H, Schroth G, Luo S, Clark DS, Chen F, Zhang T, Mackie RI, Pennacchio LA, Tringe SG, Visel A, Woyke T, Wang Z and Rubin EM 2011. Metagenomic discovery of biomass-degrading genes and genomes from cow rumen. Science 331, 463-467.

Hillman K, Lloyd D and Williams AG 1985. Use of a portable quadrupole mass spectrometer for the measurement of dissolved gas concentrations in ovine rumen liquor in situ. Current Microbiology 12, 335-339.

Hosokawa M, Nishikawa Y, Kogawa M and Takeyama H 2017. Massively parallel whole genome amplification for single-cell sequencing using droplet microfluidics. Scientific Reports 7, 5199. doi: 10.1038/s41598-017-05436-4.

Hungate RE, Reichl J and Prins R 1971. Parameters of rumen fermentation in a continuously fed sheep: evidence of a microbial rumination pool. Applied Microbiology 22, 1104-1113.

Huws SA, Creevey CJ, Oyama LB, Mizrahi I, Denman SE, Popova M, MuñozTamayo R, Forano E, Waters SM, Hess M, Tapio I, Smidt H, Krizsan SJ, Yáñez-Ruiz DR, Belanche A, Guan L, Gruninger RJ, McAllister TA, Newbold CJ, Roehe R, Dewhurst RJ, Snelling TJ, Watson M, Suen G, Hart EH, Kingston-Smith AH, Scollan ND, do Prado RM, Pilau EJ, Mantovani HC, Attwood GT, Edwards JE, McEwan NR, Morrisson S, Mayorga OL, Elliott C and Morgavi DP 2018. Addressing global ruminant agricultural challenges through understanding the rumen microbiome: past, present, and future. Frontiers in Microbiology 9, 2161. doi: 10.3389/fmicb.2018.02161.

Huws SA, Edwards JE, Creevey CJ, Rees Stevens P, Lin W, Girdwood SE, Pachebat JA and Kingston-Smith AH 2016. Temporal dynamics of the metabolically active rumen bacteria colonizing fresh perennial ryegrass. FEMS Microbiology Ecology 92, fiv137. doi: 10.1093/femsec/fiv137.

Huws SA, Mayorga OL, Theodorou MK, Kim EJ, Cookson AH, Newbold CJ and Kingston-Smith AH 2014. Differential colonization of plant parts by the rumen microbiota is likely to be due to different forage chemistries. Journal of Microbial and Biochemical Technology 6, 80-86.

Jami E, Israel A, Kotser A and Mizrahi I 2013. Exploring the bovine rumen bacterial community from birth to adulthood. The ISME Journal 7 , 1069-1079.

Janssen PH and Kirs M 2008. Structure of the archaeal community of the rumen. Applied and Environmental Microbiology 74, 3619-3625.

Jiao J, Lu Q, Forster RJ, Zhou C, Wang M, Kang J and Tan Z 2016. Age and feeding system (supplemental feeding versus grazing) modulates colonic bacterial succession and host mucosal immune maturation in goats. Journal of Animal Science 94, 2506-2518.

Kamke J, Kittelmann S, Soni P, Li Y, Tavendale M, Ganesh S, Janssen PH, Shi W, Froula J, Rubin EM and Attwood GT 2016. Rumen metagenome and metatranscriptome analyses of low methane yield sheep reveals a Sharpea-enriched 
microbiome characterised by lactic acid formation and utilisation. Microbiome 4,56 .

Khafipour E, Li S, Plaizier JC and Krause DO 2009. Rumen microbiome composition determined using two nutritional models of subacute ruminal acidosis. Applied and Environmental Microbiology 75, 7115-7124.

Khafipour E, Plaizier JC, Aikman PC and Krause DO 2011. Population structure of rumen Escherichia coli associated with subacute ruminal acidosis (SARA) in dairy cattle. Journal of Dairy Science 94, 351-360.

Kim M and Yu Z 2014. Variations in 16S rRNA-based microbiome profiling between pyrosequencing runs and between pyrosequencing facilities. The Journal of Microbiology 52, 355. doi: 10.1007/s12275-014-3443-3.

Kittelmann S, Kirk MR, Jonker A, McCulloch A and Janssen PH 2015. Buccal swabbing as a noninvasive method to determine bacterial, archaeal, and eukaryotic microbial community structures in the rumen. Applied and Environmental Microbiology 81, 7470-7483.

Koetschan C, Kittelmann S, Lu J, Al-Halbouni D, Jarvis GN, Müller T, Wolf M and Janssen PH 2014. Internal transcribed spacer 1 secondary structure analysis reveals a common core throughout the anaerobic fungi (Neocallimastigomycota). PLoS One 9, e91928.

Kong Y, Teather R and Forster R 2010. Composition, spatial distribution, and diversity of the bacterial communities in the rumen of cows fed different forages. FEMS Microbiology Ecology 74, 612-622.

Kumar S, Indugu N, Vecchiarelli B and Pitta DW 2015. Associative patterns among anaerobic fungi, methanogenic archaea, and bacterial communities in response to changes in diet and age in the rumen of dairy cows. Frontiers in Microbiology 6, 781. doi: 10.3389/fmicb.2015.00781.

Levy B and Jami E 2018. Exploring the prokaryotic community associated with the rumen ciliate protozoa population. Frontiers in Microbiology 9, 2526. doi: 10.3389/fmicb.2018.02526

Ley RE, Lozupone CA, Hamady M, Knight R and Gordon JI 2008. Worlds within worlds: evolution of the vertebrate gut microbiota. Nature Reviews Microbiology 6, 776-788.

Li F and Guan LL 2017. Metatranscriptomic profiling reveals linkages between the active rumen microbiome and feed efficiency in beef cattle. Applied and Environmental Microbiology 83, e00061-17.

Li F, Henderson G, Sun X, Cox F, Janssen PH and Guan LL 2016. Taxonomic assessment of rumen microbiota using total RNA and targeted amplicon sequencing approaches. Frontiers in Microbiology 7, 987. doi: 10.3389/fmicb. 2016.00987

Liggenstoffer AS, Youssef NH, Couger M and Elshahed MS 2010. Phylogenetic diversity and community structure of anaerobic gut fungi (phylum Neocallimastigomycota) in ruminant and non-ruminant herbivores. The ISME Journal 4, 1225-1235. doi: 10.1038/ismej.2010.49.

Liu Z, Lozupone C, Hamady M, Bushman FD and Knight R 2007. Short pyrosequencing reads suffice for accurate microbial community analysis. Nucleic Acids Research 35, e120.

Liu J, Zhang M, Xue C, Zhu W and Mao S 2016. Characterization and comparison of the temporal dynamics of ruminal bacterial microbiota colonizing rice straw and alfalfa hay within ruminants. Journal of Dairy Science 99, 9668-9681.

Liu JH, Bian GR, Zhu WY and Mao SY 2015. High-grain feeding causes strong shifts in ruminal epithelial bacterial community and expression of Toll-like receptor genes in goats. Frontiers in Microbiology 6, 167. doi: 10.3389/fmicb. 2015.00167

Mayorga OL, Kingston-Smith AH, Kim EJ, Allison GG, Wilkinson TJ, Hegarty MJ, Theodorou MK, Newbold CJ and Huws SA 2016. Temporal metagenomic and metabolomic characterization of fresh perennial ryegrass degradation by rumen bacteria. Frontiers in Microbiology 7, 1854. doi: 10.3389/fmicb.2016.01854.

McCann JC, Luan S, Cardoso FC, Derakhshani H, Khafipour E and Loor JJ 2016. Induction of subacute ruminal acidosis affects the ruminal microbiome and epithelium. Frontiers in Microbiology 7, 701. doi: 10.3389/fmicb.2016.00701.

Meale SJ, Li S, Azevedo P, Derakhshani H, Plaizier JC, Khafipour E and Steele MA 2016. Development of ruminal and fecal microbiomes are affected by weaning but not weaning strategy in dairy calves. Frontiers in Microbiology 7, 582. doi: 10.3389/fmicb.2016.00582.

Min BR, Pinchak WE, Anderson RC, Fulford JD and Puchala R 2006. Effects of condensed tannins supplementation level on weight gain and in vitro and in vivo bloat precursors in steers grazing winter wheat. Journal of Animal Science 84, 2546-2554.
Morgavi DP, Kelly WJ, Janssen PH and Attwood GT 2013. Rumen microbial (meta)genomics and its application to ruminant production. Animal 7 Suppl 1 184-201.

Myer PR, Kim M, Freetly HC and Smith TP 2016. Evaluation of 16S rRNA amplicon sequencing using two next-generation sequencing technologies for phylogenetic analysis of the rumen bacterial community in steers. Journal of Microbiological Methods 127, 132-140.

Newbold CJ, de la Fuente G, Belanche A, Ramos-Morales E and McEwan NR 2015. The role of ciliate protozoa in the rumen. Frontiers in Microbiology 6 , 1313. doi: 10.3389/fmicb.2015.01313.

Noel SJ, Attwood GT, Rakonjac J, Moon CD, Waghorn GC and Janssen PH 2017 Seasonal changes in the digesta-adherent rumen bacterial communities of dairy cattle grazing pasture. PLOS One 12, e0173819.

Owens FN, Secrist DS, Hill WJ and Gill DR 1998. Acidosis in cattle: a review. Journal of Animal Science 76, 275-286.

Petri RM, Schwaiger T, Penner GB, Beauchemin KA, Forster RJ, McKinnon JJ and McAllister TA 2013. Characterization of the core rumen microbiome in cattle during transition from forage to concentrate as well as during and after an acidotic challenge. PLoS One 8, e83424.

Piao H, Lachman M, Malfatti S, Sczyrba A, Knierim B, Auer M, Tringe SG, Mackie RI, Yeoman CJ and Hess M 2014. Temporal dynamics of fibrolytic and methanogenic rumen microorganisms during in situ incubation of switchgrass determined by $16 \mathrm{~S}$ rRNA gene profiling. Frontiers in Microbiology 5, 307. doi: 10 . 3389/fmicb.2014.00307.

Pitta DW, Pinchak WE, Dowd S, Dorton K, Yoon I, Min BR, Fulford JD, Wickersham TA and Malinowski DP 2014. Longitudinal shifts in bacterial diversity and fermentation pattern in the rumen of steers grazing wheat pasture. Anaerobe 30, 11-17

Pitta DW, Pinchak WE, Indugu N, Vecchiarelli B, Sinha R and Fulford JD 2016. Metagenomic analysis of the rumen microbiome of steers with wheat-induced frothy bloat. Frontiers in Microbiology 7, 689. doi: 10.3389/fmicb.2016.00689. Plaizier JC, Li S, Danscher AM, Derakshani H, Andersen PH and Khafipour E 2017. Changes in microbiota in rumen digesta and feces due to a grain-based subacute ruminal acidosis (SARA) challenge. Microbial Ecology 74, 485-495.

Plaizier JC, Li S, Tun HM and Khafipour E 2016. Nutritional models of experimentally-induced subacute ruminal acidosis (SARA) differ in their impact on rumen and hindgut bacterial communities in dairy cows. Frontiers in Microbiology 7 , 2128.

Pollock J, Glendinning L, Wisedchanwet T and Watson M 2018. The madness of microbiome: attempting to find consensus 'Best Practice' for $16 \mathrm{~S}$ microbiome studies. Applied and Environmental Microbiology 84, e02627-17.

Qi M, Wang P, O'Toole N, Barboza PS, Ungerfeld E, Leigh MB, Selinger LB, Butler $G$, Tsang A and McAllister TA 2011. Snapshot of the eukaryotic gene expression in muskoxen rumen - a metatranscriptomic approach. PLoS One 6, e20521.

Ribeiro GO,Gruninger RJ, Badhan A and McAllister TA 2016. Mining the rumen for fibrolytic feed enzymes. Animal Frontiers 6, 20-26.

Ribeiro GO, Oss DB, He Z, Gruninger RJ, Elekwachi C, Forster RJ, Yang W, Beauchemin KA and McAllister TA 2017. Repeated inoculation of cattle rumen with bison rumen contents alters the rumen microbiome and improves nitrogen digestibility in cattle. Scientific Reports 7, 1276.

Rubino F, Carberry C, Waters SM, Kenny D, McCabe MS and Creevey CJ 2017. Divergent functional isoforms drive niche specialisation for nutrient acquisition and use in rumen microbiome. The ISME Journal 11, 2538-2551.

Schloss PD, Jenior ML, Koumpouras CC, Westcott SL and Highlander SK 2016. Sequencing 16S rRNA gene fragments using the PacBio SMRT DNA sequencing system. PeerJ 4, e1869.

Seshadri R, Leahy SC, Attwood GT, Teh KH, Lambie SC, Cookson AL, EloeFadrosh EA, Pavlopoulos GA, Hadjithomas M, Varghese NJ, Paez-Espino D, Hungate 1000 project collaborators, Perry $R$, Henderson $G$, Creevey $C_{\text {, }}$ Terrapon N, Lapebie P, Drula E, Lombard V, Rubin E, Kyrpides NC, Henrissat B, Woyke T, Ivanova NN and Kelly WJ 2018. Cultivation and sequencing of rumen microbiome members from the Hungate1000 Collection. Nature Biotechnology 36, 359-367.

Shaani Y, Zehavi T, Eyal S, Miron J and Mizrahi I 2018. Microbiome niche modification drives diurnal rumen community assembly, overpowering individual variability and diet effects. The ISME Journal 12, 2446-2457. doi: 10.1038/ s41396-018-0203-0.

Shabat SK, Sasson G, Doron-Faigenboim A, Durman T, Yaacoby S, Berg Miller ME, White BA, Shterzer N and Mizrahi I 2016. Specific microbiome-dependent 


\section{Gruninger, Ribeiro, Cameron and McAllister}

mechanisms underlie the energy harvest efficiency of ruminants. The ISME Journal 10, 2958-2972. doi:10.1038/ismej.2016.62.

Shinkai T, Mitsumori $M$, Sofyan A, Kanamori $H$, Sasaki $H$, Katayose $Y$ and Takenaka A 2016. Comprehensive detection of bacterial carbohydrate-active enzyme coding genes expressed in cow rumen. Animal Science Journal 87, 1363-1370.

Soergel DA, Dey N, Knight R and Brenner SE 2012. Selection of primers for optimal taxonomic classification of environmental 16S rRNA gene sequences. The ISME Journal 6, 1440. doi: 10.1038/ismej.2011.208.

Solomon KV, Haitjema CH, Henske JK, Gilmore SP, Borges-Rivera D, Lipzen A Brewer HM, Purvine SO, Wright AT, Theodorou MK, Grigoriev IV, Regev A Thompson DA, and O'malley MA 2016. Early-branching gut fungi possess a large, comprehensive array of biomass-degrading enzymes. Science 351, 1192-1195.

Stewart RD, Auffret MD, Warr A, Wiser AH, Press MO, Langford KW, Liachko I, Snelling TJ, Dewhust RJ, Walker AW, Roche R, and Watson M 2018a. Assembly of 913 microbial genomes from metagenomic sequencing of the cow rumen. Nature Communications 9, 870

Stewart RD, Auffret MD, Warr A, Wiser AH, Walker AW and Watson M 2018b. The genomic and proteomic landscape of the rumen microbiome revealed by comprehensive genome-resolved metagenomics. bioRxiv http://dx.doi.org/10. $1101 / 489443$.

Svartström 0, Alneberg J, Terrapon N, Lombard V, de Bruijn I, Malmsten J, Dalin A-M, El Muller E, Shah P and Wilmes P 2017. Ninety-nine de novo assembled genomes from the moose (Alces alces) rumen microbiome provide new insights into microbial plant biomass degradation. The ISME Journal 11, 2538-2551.

Tapio I, Fischer D, Blasco L, Tapio M, Wallace RJ, Bayat AR, Ventto L, Kahala M Negussie E and Shingfield KJ 2017. Taxon abundance, diversity, co-occurrence and network analysis of the ruminal microbiota in response to dietary changes in dairy cows. PLoS One 12, e0180260.

Taxis TM, Wolff S, Gregg SJ, Minton NO, Zhang C, Dai J, Schnabel RD, Taylor JF, Kerley MS, Pires JC, Lamberson WR and Conant GC 2015. The players may change but the game remains: network analyses of ruminal microbiomes suggest taxonomic differences mask functional similarity. Nucleic Acids Research 43, 9600-9612.

Tremblay J, Singh K, Fern A, Kirton ES, He S, Woyke T, Lee J, Chen F, Dangl JL and Tringe SG 2015. Primer and platform effects on 16S rRNA tag sequencing Frontiers in Microbiology 6, 771. doi: 10.3389/fmicb.2015.00771.
Valle ER, Henderson G, Jansen PH, Cox F, Alexander TW and McAllister TA 2015 Considerations in the use of flourescence in situ hybridization (FISH) and confocal lazer scanning microscopy to characterize rumen methogens and defime their spatial distribution. Canadian Journal of Microbiology 61, 417-428.

Wang L, Xu Q, Kong F, Yang Y, Wu D, Mishra S and Li Y 2016a. Exploring the goat rumen microbiome from seven days to two years. PLoS One 11, e0154354.

Wang PP, Zhao SG, Wang XW, Zhang YD, Zheng N, and Wang JQ 2016b. Ruminal methanogen community in dairy cows fed agricultural residues of corn stover, rapeseed and cottonseed meals. Journal of Agricultural and Food Chemistry 64, 5439-5445.

Wang Z, Elekwachi CO, Jiao J, Wang M, Tang S, Zhou C, Tan Z and Forster RJ 2017. Investigation and manipulation of metabolically active methanogen community composition during rumen development in black goats. Scientific Reports 7, 422. doi: 10.1038/s41598-017-00500-5.

Weimer PJ 2015. Redundancy, resilience, and host specificity of the ruminal microbiota: implications for engineering improved ruminal fermentations. Frontiers in Microbiology 6, 296. doi: 10.3389/fmicb.2015.00296

Wenner BA, Wagner BK and Firkins JL 2018. Using video microscopy to improve quantitative estimates of protozoal motility and cell volume. Journal of Dairy Science 101, 1069-1073.

Yang B, Wang Y and Qian P-Y 2016. Sensitivity and correlation of hypervariable regions in 16S rRNA genes in phylogenetic analysis. BMC Bioinformatics 17, 135. doi: 10.1186/s12859-016-0992-y.

Yarlett N, Orpin CG, Munn EA, Yarlett NC, and Greenwood CA 1986 Hydrogenosomes in the rumen fungus Neocallimastix patriciarum. Biochemica Journal 236, 729-739.

Youssef NH, Couger MB, Struchtemeyer CG, Liggenstoffer AS, Prade RA, Najar FZ, Atiyeh HK, Wilkins MR, and Elshahed MS 2013. The genome of the anaerobic fungus Orpinomyces sp. strain C1A reveals the unique evolutionary history of a remarkable plant biomass degrader. Applied and Environmental Microbiology 79, 4620-4634.

Ze X, Le Mougen F, Duncan SH, Louis P and Flint HJ 2013. Some are more equal than others: the role of 'keystone' species in the degradation of recalcitrant substrates. Gut Microbes 4, 236-240.

Zened A, Combes S, Cauquil L, Mariette J, Klopp C, Bouchez O, TroegelerMeynadier A and Enjalbert F 2013. Microbial ecology of the rumen evaluated by 454 GS FLX pyrosequencing is affected by starch and oil supplementation of diets. FEMS Microbiology Ecology 83, 504-514. 